\title{
On the relation between the non-flat cosmological models and the elliptic integral of first kind (Research Note)
}

\author{
A. Mészáros ${ }^{1}$ and J. Řípa ${ }^{2}$ \\ ${ }^{1}$ Charles University in Prague, Faculty of Mathematics and Physics, Astronomical Institute, V Holešovičkách 2, 18000 Prague 8, \\ Czech Republic \\ e-mail: meszaros@cesnet.cz \\ 2 Institute of Basic Science, Natural Sciences Campus, Sungkyunkwan University, Engineering Building 2, 2066 Seobu-ro, \\ Jangan-gu, Suwon, 440-746 Gyeonggi-do, Korea \\ e-mail: ripa.jakub@gmail.com
}

Received 22 October 2014 / Accepted 25 October 2014

\section{ABSTRACT}

Context. Recently we have found an analytic integration formula that describes the dependence of the luminosity distance on the redshift for the flat cosmological model with a non-zero cosmological constant.

Aims. The purpose of this article is to search for a similar relation for the non-flat models.

Methods. A Taylor series was used.

Results. The elliptic integral of the first kind can indeed be applied.

Conclusions. The result shows that a combination of the numerical integration with the previous analytic formula can also be useful for the nearly flat cosmological models.

Key words. cosmology: theory

\section{Introduction}

In our recent paper (Mészáros \& Řípa 2013), we have shown that the standard cosmological dependence of the luminosity distance $d_{\mathrm{L}}(z)$ on the redshift $z$ can also be described analytically for a non-zero cosmological constant, especially if the Universe is spatially flat. In this result the elliptic integral of the first kind plays a key role.

The question emerges immediately of whether the restriction to the flat cosmological model is needed or does the elliptic integral of the first kind play a role also for non-flat models with nonzero cosmological constant? This note studies these questions.

\section{A recapitulation of the known relations}

We start with (Carroll et al. 1992)

$$
\begin{aligned}
& d_{\mathrm{PM}}(z)=\frac{c}{H_{0} \sqrt{\left|\Omega_{k}\right|}} \\
& \times \operatorname{sinn}\left\{\sqrt{\left|\Omega_{k}\right|} \int_{0}^{z} \frac{\mathrm{d} z^{\prime}}{\sqrt{\left(1+z^{\prime}\right)^{3} \Omega_{\mathrm{M}}+\left(1+z^{\prime}\right)^{2} \Omega_{k}+\Omega_{\Lambda}}}\right\} .
\end{aligned}
$$

In this equation, $c$ is the speed of light in vacuum, $H_{0}$ is the Hubble-constant, and $\Omega_{k}+\Omega_{\mathrm{M}}+\Omega_{\Lambda}=1$. The notation "sinn" means the standard function sinh for $\Omega_{k}>0$, and $\sin$ for $\Omega_{k}<0$, respectively. If $\Omega_{k}=0$, then "sinn $x$ " means $x$ and one simply has

$$
\frac{H_{0} d_{\mathrm{PM}}(z)}{c}=\int_{0}^{z} \frac{\mathrm{d} z^{\prime}}{\sqrt{\left(1+z^{\prime}\right)^{3} \Omega_{\mathrm{M}}+\Omega_{\Lambda}}},
$$

where $\Omega_{\mathrm{M}}+\Omega_{\Lambda}=1$. The proper-motion $d_{\mathrm{PM}}(z)$ distance is connected to luminosity distance $d_{\mathrm{L}}(z)$ by relation $d_{\mathrm{PM}}(z)(1+z)=$ $d_{\mathrm{L}}(z)$ (Weinberg 1972). In both equations, it must be $\Omega_{\mathrm{M}} \geq 0$. (The case $\Omega_{M}=0$ is unphysical, but it can serve as a limit.) The remaining two omega factors can have both signs.

Equation (1) of Mészáros \& Řípa (2013) and Eq. (1) here are equivalent. Equation (3) of Mészáros \& Řípa (2013) and Eq. (2) here are identical.

In the special case of $\Omega_{\Lambda}=0$, the integral in Eq. (1) can be evaluated by the so-called Mattig formula (Mattig 1958). In the special case of $\Omega_{k}=0$, the elliptic integrals can be used (Mészáros \& Řípa 2013). The special case, where $\Omega_{\mathrm{M}}=0$, in Eq. (1) the integral is very simple. As a result, if one omega factor is zero, but the remaining two ones are non-zeros, no numerical integration is needed. In addition, if just two omega factors are simultaneously zeros, then the integration via primitive functions is very easy. Only the case where all three omega factors are non-zeros needs numerical integration. We also search for eventual analytical integrations here.

\section{Integration}

In what follows we assume that $\Omega_{\Lambda} \neq 0, \Omega_{k} \neq 0, \Omega_{\mathrm{M}}>0$, and $\Omega_{k}+\Omega_{\mathrm{M}}+\Omega_{\Lambda}=1$ in Eq. (1). We rewrite the denominator of Eq. (1) as follows:

$$
\begin{aligned}
& \sqrt{\left(1+z^{\prime}\right)^{3} \Omega_{\mathrm{M}}+\left(1+z^{\prime}\right)^{2} \Omega_{k}+\Omega_{\Lambda}}= \\
& \sqrt{\left(1+z^{\prime}\right)^{3} \Omega_{\mathrm{M}}+\Omega_{\Lambda}} \times \sqrt{1+\frac{\left(1+z^{\prime}\right)^{2} \Omega_{k}}{\left(1+z^{\prime}\right)^{3} \Omega_{\mathrm{M}}+\Omega_{\Lambda}}} .
\end{aligned}
$$


Using only the first two terms in the Taylor series for function $g(Q)$,

$g(Q)=\frac{1}{\sqrt{1+Q}}=1-\frac{Q}{2}+\ldots$

and defining

$$
Q=\frac{\left(1+z^{\prime}\right)^{2} \Omega_{k}}{\left(1+z^{\prime}\right)^{3} \Omega_{\mathrm{M}}+\Omega_{\Lambda}},
$$

we obtain for the integral in Eq. (1)

$$
\begin{aligned}
\int_{0}^{z} & \frac{\mathrm{d} z^{\prime}}{\sqrt{\left(1+z^{\prime}\right)^{3} \Omega_{\mathrm{M}}+\left(1+z^{\prime}\right)^{2} \Omega_{k}+\Omega_{\Lambda}}}= \\
& \int_{0}^{z} \frac{\mathrm{d} z^{\prime}}{\sqrt{\left(1+z^{\prime}\right)^{3} \Omega_{\mathrm{M}}+\Omega_{\Lambda}}} \\
& -\Omega_{k} \int_{0}^{z} \frac{\left(1+z^{\prime}\right)^{2} \mathrm{~d} z^{\prime}}{2\left(\Omega_{\mathrm{M}}\left(1+z^{\prime}\right)^{3}+\Omega_{\Lambda}\right)^{3 / 2}}+\ldots
\end{aligned}
$$

It is essential to emphasize here that the first term on the right hand side of this equation is not identical to the right hand side of Eq. (2). The difference is given by the fact that in Eq. (2) it holds that $\Omega_{\mathrm{M}}+\Omega_{\Lambda}=1$, but here it holds that $\Omega_{\mathrm{M}}+\Omega_{\Lambda}=1-\Omega_{k} \neq 1$.

Inspecting Eqs. (3) -(13) of Mészáros \& Řípa (2013), we recognize that the constraint $\Omega_{\mathrm{M}}+\Omega_{\Lambda}=1$ is not needed from the mathematical point of view. We can easily repeat the whole integration procedure of Eqs. (3)-(13) in Mészáros \& Řípa (2013) for any $\Omega_{\mathrm{M}}>0$ and $\Omega_{\Lambda}>0$. All this means that the first term on the right hand side of Eq. (6) can again be solved analytically using the elliptic integral of first kind. The only difference is given in Eq. (5) of Mészáros \& Řípa (2013). In Mészáros \& Řípa (2013) $\Omega_{\mathrm{M}}+\Omega_{\Lambda}=1$ was used, but here both omega factors can be arbitrarily positive. This means that only the factor $\Omega_{M}^{1 / 3} \Omega_{\Lambda}^{1 / 6}$ is influenced, but the integral in Eq. (5) of Mészáros \& Řípa (2013) itself remains unchanged. For the sake of completeness, it must be added that, using the remark of Sect. 3 of Mészáros \& Řípa (2013), we can generalize here the solution also for arbitrary $\Omega_{\Lambda}<0$ and $\Omega_{M}>0$. Hence, the first term of the right hand side of Eq. (6) can always be calculated analytically.

On the other hand, the second term of Eq. (6) is a complicated formula, and we did not find any primitive function for this integral. It should therefore be calculated numerically. In either case, this integral is finite for any $0<z<\infty$.

\section{Remarks}

It may seem that Eq. (6) did not give any new result, because in essence - some numerical integration is again necessary. But this is not the case, because for small $0<\left|\Omega_{k}\right| \ll 1 \simeq \Omega_{\mathrm{M}}+\Omega_{\Lambda}$, the procedure of the previous section can be useful. This can be seen as follows.
The interval of convergence of the Taylor series for $g(Q)$ in Eq. (4) is given by $|Q|<1$. This means that it has to be

$0<\left|\left(1+z^{\prime}\right)^{3} \Omega_{\mathrm{M}}+\Omega_{\Lambda}\right|-\left(1+z^{\prime}\right)^{2}\left|\Omega_{k}\right|=f\left(z^{\prime}\right)$

for any $\infty>z^{\prime} \geq 0$. If this requirement holds, then even higher terms of the Taylor series can be used.

The requirement of Eq. (7) can also be formulated in another form that does not use $z^{\prime}$. For $-\Omega_{\Lambda} \geq \Omega_{\mathrm{M}}>0$, the first term in $f\left(z^{\prime}\right)$ is zero for $1+z^{\prime}=\left(-\Omega_{\Lambda} / \Omega_{\mathrm{M}}\right)^{1 / 3} \geq 1$. Hence, for this $z^{\prime}$ one has $f\left(z^{\prime}\right)<0$, and $|Q|<1$ is not fulfilled. Thus, the term $\left(1+z^{\prime}\right)^{3} \Omega_{\mathrm{M}}+\Omega_{\Lambda}$ must be positive. For $z^{\prime}=0$ we must also have $|Q|<1$, and it has to be $\Omega_{\mathrm{M}}+\Omega_{\Lambda}>\left|\Omega_{k}\right|$. This combination of the omega factors is thus the necessary condition for $|Q|<1$. To obtain the sufficient condition, one has to discuss $f\left(z^{\prime}\right)$ as a standard cubic function. If $2\left|\Omega_{k}\right| /\left(3 \Omega_{\mathrm{M}}\right)<1$ holds, then the necessary condition is also a sufficient one, because then $f\left(z^{\prime}\right)$ is an increasing function for any $z^{\prime} \geq 0$. If $2\left|\Omega_{k}\right| /\left(3 \Omega_{\mathrm{M}}\right) \geq 1$ holds, then one must have $f\left(z^{\prime}\right)>0$ for $1+z^{\prime}=2\left|\Omega_{k}\right| /\left(3 \Omega_{\mathrm{M}}\right)$, because $f\left(z^{\prime}\right)$ has a minimum for this $z^{\prime}$. It is positive for $\Omega_{\Lambda}>$ $4\left|\Omega_{k}\right|^{3} /\left(27 \Omega_{\mathrm{M}}^{2}\right)$ and $|Q|<1$ holds; but it is non-positive for $\Omega_{\Lambda} \leq$ $4\left|\Omega_{k}\right|^{3} /\left(27 \Omega_{\mathrm{M}}^{2}\right)$ and $|Q|<1$ does not hold.

All this means that mainly for $0<\left|\Omega_{k}\right| \ll \Omega_{\mathrm{M}}+\Omega_{\Lambda} \simeq 1$ the procedure of this note is quite usable. (For the sake of precision it must be added that either the condition $2\left|\Omega_{k}\right| /\left(3 \Omega_{\mathrm{M}}\right)<1$ or the condition $\Omega_{\Lambda}>4\left|\Omega_{k}\right|^{3} /\left(27 \Omega_{\mathrm{M}}^{2}\right)$ must also be fulfilled.) In this case, the second term in Eq. (6) is a small correction to the first one. Then it can be simpler to numerically calculate only this small correction and not the whole integral of Eq. (1). In any case, our "semi-analytical" procedure can serve as a check of the numerical calculation of Eq. (1).

\section{Conclusion}

We have shown that the integral on the right hand side of Eq. (1) can be partly calculated analytically using the elliptic integral of the first kind even in the case, when all the three omega factors are non-zeros. This calculation can be useful for $0<\left|\Omega_{k}\right| \ll 1$.

Acknowledgements. We wish to thank A. Cappi, D. Eisenstein, B. Fulford, M. Křížek, and N. Ryan for useful discussions. This study was supported by the Grant Agency of the Czech Republic Grant P209/10/0734, and by Creative Research Initiatives (RCMST) of MEST/NRF.

\section{References}

Carroll, S. M., Press, W. H., \& Turner, E. L. 1992, ARA\&A, 30, 499 Mattig, W. 1958, Astron. Nachr., 284, 109

Mészáros, A., \& Řípa, J. 2013, A\&A, 556, A13

Weinberg, S. 1972, Gravitation and Cosmology: Principles and Applications of the General Theory of Relativity (Wiley) 